\title{
The unique aspects of the nutrition transition in South Korea: the retention of healthful elements in their traditional diet
}

\author{
Min-June Lee ${ }^{1, *}$, Barry M Popkin ${ }^{2}$ and Soowon Kim ${ }^{3}$ \\ ${ }^{1}$ Research Institute of Food and Nutritional Sciences, Yonsei University, 134 Shinchon-dong Sodaemoon-gu, Seoul \\ 120-749, South Korea: ${ }^{2}$ University of North Carolina at Chapel Hill, Carolina Population Center, CB \# 8120 \\ University Square, 123 West Franklin Street, Chapel Hill, NC 27516-3997, USA: ${ }^{3}$ University of North Carolina at \\ Chapel Hill, Department of Nutrition, Chapel Hill, NC 27516, USA
}

\begin{abstract}
Objective: The purposes of this paper are to describe the unique aspects of the nutrition transition in South Korea, including trends in food consumption and obesity, patterns of morbidity and mortality; to focus on efforts to maintain the traditional diet in the midst of rapid economic growth and the introduction of Western culture; and to provide insights for other countries.

Design: We analysed secondary dietary intake, anthropometric, morbidity and mortality data from published reports and articles.

Results: In South Korea, the level and rate of increase in fat intake have remained very low, whereas vegetable intake has been high and fruit intake has increased greatly. South Korea also has a relatively low prevalence of obesity compared with other Asian countries. The traditional Korean diet is a low-fat and high-vegetable diet. Therefore, the government and nutrition specialists have been initiating numerous efforts to advertise and teach the public that the traditional diet is a healthy diet. They are also working on revival of the traditional diet using an approach that is acceptable to contemporary Koreans.

Conclusions: The nutrition transition in South Korea is unique. A range of government, nutrition specialists and some private organisation efforts has worked to retain healthful elements of the traditional diet in South Korea. The continued low level of total fat in the overall diet and the high intake of fruits and vegetables bode well for South Korea.
\end{abstract}

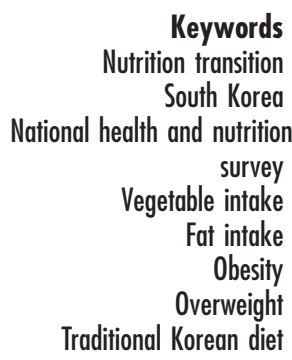

South Korea has experienced various and rapid socioeconomic changes during the past three decades. Since the first Five-year Economic Development Plan was established in 1962 to revive the economy after the Korean War, the Korean economy has grown with great rapidity. Similar to the nutrition transition in Japan, the South Korean transition has also been very rapid. However, unlike Japan and other Asian countries, South Korea has maintained many of the aspects of the traditional diet, shaping a unique transition in this country. The lower than expected levels of fat intake and obesity prevalence - based on its level of economic development - have been described as the unique aspects of the nutrition transition in South Korea ${ }^{1}$.

The purpose of this paper is to describe the unique aspects of the nutrition transition in South Korea, including trends in food consumption and obesity, as well as patterns of morbidity and mortality. We also explore reasons for the retention of the healthy elements of the traditional diet in South Korea, focusing on efforts to maintain the traditional diet in the midst of the rapid economic growth and the introduction of Western culture, and to provide insights for other countries.

\section{Subjects and methods}

We analysed secondary dietary intake, anthropometric, morbidity and mortality data from published reports and articles.

\section{Dietary data}

The National Nutrition Survey, conducted from 1969 until 1995, and the National Health and Nutrition Survey of 1998 provided the diet trends data ${ }^{2}$. The first National Health and Nutrition Survey in South Korea was created in 1998 by combining the National Nutrition Survey and the National Health Interview Survey. The survey was divided into four parts: the Health Interview Survey (HIS), the Health Examination Survey (HES), the Health Behaviour Survey (HBS) and the Nutrition Survey.

The Nutrition Survey conducted in 1998 differs from the previous national nutrition surveys held prior to 1995 in several aspects. A stratified multistage probability sampling design was used in the 1998 survey. The sample size of the HIS was 12000 households and the other three surveys (HES, HBS and Nutrition Survey) were conducted on a sub-sample of 4000 households. In the Nutrition 
Survey, 11525 members from 3799 sample households responded. Most importantly, unlike the previous surveys - in which food intake was measured at the household level by a weighed method, the 1998 Nutrition Survey assessed food consumption at an individual level by the 24-hour recall method ${ }^{1,2}$.

\section{Obesity and overweight data}

We used body mass index (BMI, in $\mathrm{kg} \mathrm{m}^{-2}$ ) data from the National Nutrition Survey in 1995 and National Health and Nutrition Survey in 1998 to determine the prevalences of overweight and obesity of the Korean people. Only the 1995 and 1998 surveys have incorporated international standards for measuring obesity and overweight. We followed the World Health Organization cut-offs to delineate obesity (BMI of $25.0-29.99 \mathrm{~kg} \mathrm{~m}^{-2}$ for overweight (pre-obesity) and $\geq 30.0 \mathrm{~kg} \mathrm{~m}^{-2}$ for obesity) ${ }^{3}$.

\section{Morbidity/mortality data}

National morbidity data for chronic conditions such as hypertension, diabetes and hyperlipidaemia were available only from the results of the 1998 National Health and Nutrition Survey $^{2}$. Therefore, we present the prevalence of these conditions in 1998 to provide a snapshot of current morbidity levels in South Korea. For mortality data, we present cause-of-death trends as the rate of mortality per 100000 of the population for four disease categories in which the death rates changed noticeably in South Korea during the past several decades ${ }^{4}$.

\section{Results}

\section{Changes in dietary intake}

The trends in intakes by major food groups and nutrients in South Korea have been described in detail by Kim et al. ${ }^{1}$. A large increase in the consumption of animal food products and a fall in total cereal intake summarise the major dietary changes in the South Korean nutrition transition. An examination of the most recent data from the 1998 Nutrition Survey reveals that the share of dairy products consumed began to exceed the consumption of fish and shellfish, which had remained the highest among animal food intakes until 1995. The main ingredient of the Korean diet can be determined by examining what foods are consumed the most in South Korea. According to the results of the 1998 Nutrition Survey, Koreans consumed 246.1 g person $^{-1}$ day $^{-1}$ of rice (the staple and most consumed food of Korea), which was $19.1 \%$ of the total amount of food consumed. The next most consumed food was Korean cabbage Kimchi $\left(83.8\right.$ g $_{\text {person }}{ }^{-1}$ day $^{-1}$ were consumed, or $6.8 \%$ of the total food consumed), followed by tangerines $(73.1 \mathrm{~g})$, milk $(71.0 \mathrm{~g})$, radishes $(40.5 \mathrm{~g})$, apples ( $40.1 \mathrm{~g}$ ), persimmons (39.8 g), pork (27.7 g), beef $(26.2 \mathrm{~g})$ and tofu $(24.3 \mathrm{~g})$. The 30 most consumed foods were similar in all areas except for rural areas, where three different kinds of Kimchi were included in the top 10 most consumed foods ${ }^{2}$.

It is clear from the examination of the most consumed foods that plant foods, including fruits and vegetables, still play the central role in the dietary intake of South Koreans. The per capita South Korean vegetable intake level of over $280 \mathrm{~g} \mathrm{day}^{-1}$ is among the highest in $\mathrm{Asia}^{5}$. This is a very unique aspect of the South Korean nutrition transition, along with the low level of fat intake described by Kim et al. ${ }^{1}$.

\section{Fruit and vegetable consumption pattern}

The daily per capita intake of fruits has been increasing; the increase has been especially rapid in the 1990s. In $1998,197.5 \mathrm{~g}$ of fruits were consumed daily per capita, and this is more than a tenfold increase from the $18.9 \mathrm{~g}$ consumed in 1970. The survey showed that the fruits eaten mainly by Koreans were apples, tangerines and persimmons. This survey was taken in November when these fruits were in season, and this probably affected the outcome of the survey. The intake of tangerines showed the most change among fruits; consumption was low up until the early 1970 s but then grew rapidly reaching $15.4 \mathrm{~g}$ in $1990,30.7 \mathrm{~g}$ in 1993 and $73.1 \mathrm{~g}$ in 1998, making it the most consumed fruit ${ }^{2}$.

The intake of vegetables did not show a distinct yearly increase or decrease but daily per capita intake is still more than $280 \mathrm{~g}$ in 1998, holding a steady level of $20 \%$ of the total food consumption, making it the first subsidiary food (Table 1). Examination of the consumption pattern of vegetables revealed that, with the exception of 1969 , different varieties of Kimchi accounted for over $40 \%$ of all vegetable intake. The proportion of Korean cabbage

Table 1 The average daily intake of vegetables (g person ${ }^{-1}$ day $\left.^{-1}\right)^{2}$

\begin{tabular}{lrrrr}
\hline & 1969 & 1980 & 1990 & $1998^{*}$ \\
\hline Korean cabbage Kimchi & 9.8 & 102.6 & 97.6 & 83.8 \\
Other Kimchi & 33.9 & 17.9 & 17.3 & 39.6 \\
Kimchi (total) & 43.7 & 120.5 & 114.9 & 123.4 \\
Radishes & 2.0 & 74.3 & 38.7 & 40.5 \\
Chinese cabbage & 2.3 & 38.2 & 14.8 & 12.8 \\
Pepper & 34.1 & 6.4 & 6.2 & 3.6 \\
Green onions & 6.3 & 13.5 & 10.8 & 13.2 \\
Garlic & 2.5 & 5.5 & 4.9 & 4.2 \\
Squash & 50.1 & 5.0 & 9.7 & 10.8 \\
Soybean sprouts & - & - & - & 13.4 \\
Spinach & - & - & 13.0 & 9.6 \\
Onions & - & - & 7.8 & 14.6 \\
Carrots & - & - & 2.2 & 5.5 \\
Lettuce & - & - & 5.5 & 2.9 \\
Cabbage & - & - & 1.0 & 3.1 \\
Cucumber & - & - & 4.7 & 4.9 \\
Other vegetables & 130.0 & 37.7 & 46.8 & 21.0 \\
Total & 271.0 & 301.1 & 281.0 & 283.5 \\
\hline
\end{tabular}

*Data from 1969-1995 were formed on the household-based weighing method, data from 1998 were individually based on the 24-hour recall method. 


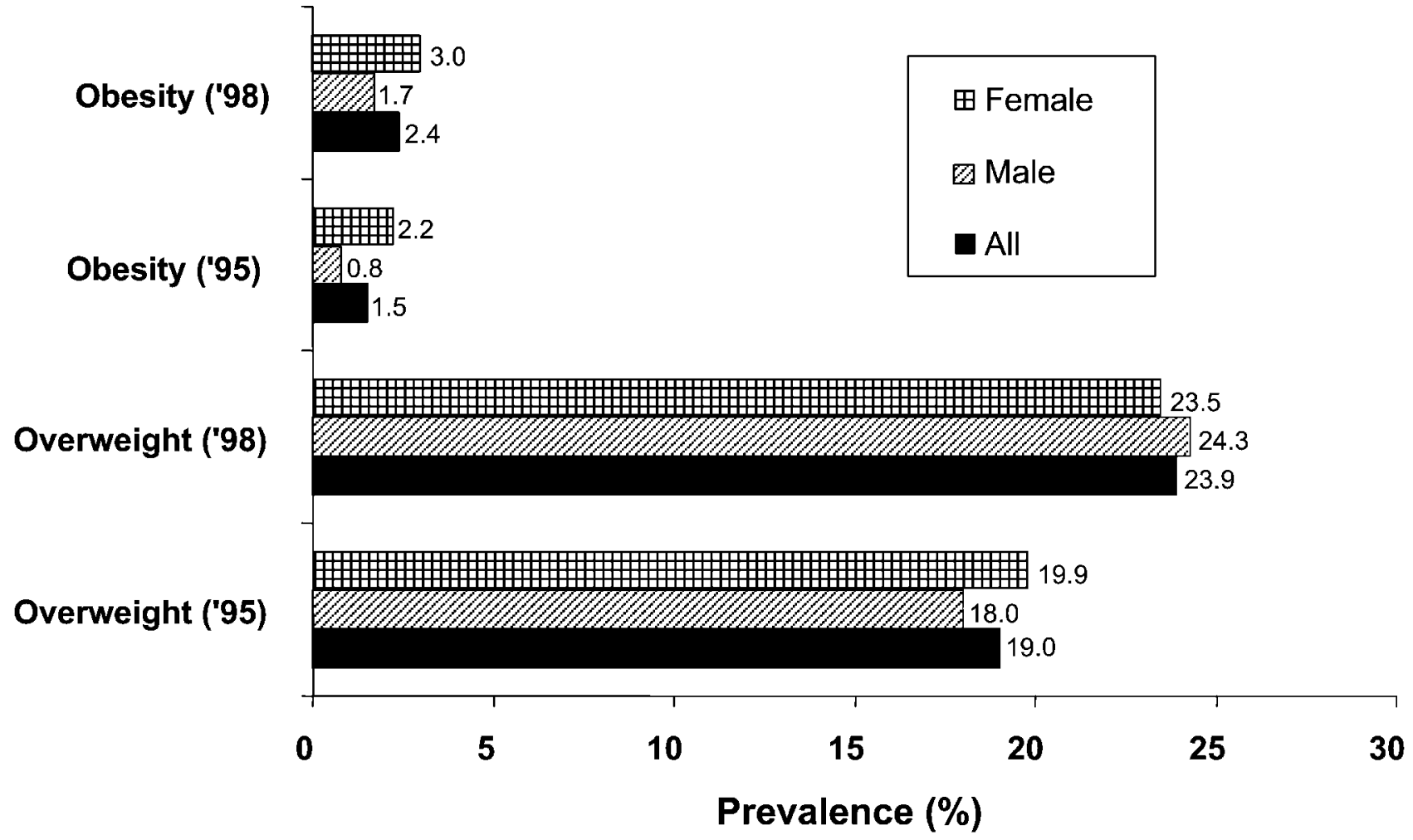

Fig. 1 Prevalence of overweight and obesity among adults in South Korea ${ }^{2}$

Kimchi consumption among the total vegetable intake has been the highest, even though it has shown a steady decrease. Excluding Kimchi consumption, the consumption of radishes, Chinese cabbage and condiments such as garlic and green onions was significant among vegetables consumed. However, given the fact that radishes, Chinese cabbage, green onions and garlic are the main ingredients of Kimchi, it is easy to see how important these four vegetables are to the Korean diet. With the addition of onions, soybean sprouts, squash and spinach, these vegetables compose $80 \%$ of the total vegetable intake. There is a systematic variation in vegetable consumption patterns shown in the surveys due to their timing. For example, the results of the 1969 survey showed that the consumption of Kimchi, radishes, Chinese cabbage, green onions and garlic was very low, whereas that of squash and pepper was very high. This reflects a seasonal variation in the vegetable consumption pattern, rather than a real change in the consumption trends. The kinds of vegetables consumed became more diverse after 1990.

\section{Fat intake pattern}

Daily per capita fat intake in South Korea more than doubled from $16.9 \mathrm{~g}$ in 1969 to $41.5 \mathrm{~g}$ in 1998. Fat intakes derived from plant foods and animal foods are both increasing. However, the animal fat share among total fat intake has increased from 30.6\% in 1970 to $48.2 \%$ in 1998, while the plant share of fat intake has decreased gradually from $70 \%$ in the 1970 s to approximately $50 \%$ in the $1990 \mathrm{~s}^{2}$. Although fat intake in South Korea is gradually rising, the proportion of fat-derived energy is significantly lower than in other Asian countries ${ }^{1}$.

After the 1990s, fat obtained from meat and meat products began to predominate in the South Korean diet. Fat from pork products represented $13.7 \%$ of the total amount of fat consumed in 1998. In the early 1990s and afterwards, beef, soybean oil, rice, ramen, eggs, milk, confectioneries and baked goods, and sesame oil followed pork as the main sources ${ }^{2,6}$. There was a decline in the proportion of vegetable oils and fats among total fat intake, decreasing from $19.4 \%$ of fat in 1990 to $13.7 \%$ in 1998. Again, this is important and contrasts with the rapid increase in edible vegetable oil in other Asian countries.

\section{Prevalence of obesity and overweight}

South Korea has a remarkably low level of adult obesity, but a higher level of overweight (Fig. 1). The 1998 survey showed that the obesity rate among adults was $1.7 \%$ for men and $3.0 \%$ for women, which was an increase of 0.9 percentage point for men (more than doubling) and 0.8 percentage point for women from 1995 . The prevalence of overweight in 1998 was $24.3 \%$ and $23.5 \%$ for men and women, respectively. This is an increase of 6.3 percentage points for men and 3.6 percentage points for women in three years. Increases in the prevalence of both obesity and overweight were greater among men. As shown in 
Table 2 Prevalence of obesity (\%)*

\begin{tabular}{lccc}
\hline & \multicolumn{3}{c}{ Age (years) } \\
\cline { 2 - 4 } & $19-44$ & $45-64$ & $>65$ \\
\hline All & 2.19 & 2.56 & 2.48 \\
Area & & & \\
$\quad$ Urban & 1.83 & 2.45 & 3.23 \\
$\quad$ Rural & 4.26 & 2.88 & 1.51 \\
Gender & & & \\
$\quad$ Male & 2.03 & 1.25 & 0.00 \\
$\quad$ Female & 2.35 & 3.82 & 3.98 \\
Income level (Won) & & & \\
$\quad<500$ 000 & 2.20 & 2.90 & 2.52 \\
$\quad 510000-1500000$ & 2.54 & 2.35 & 3.25 \\
$\quad>1510000$ & 1.69 & 2.64 & 0.90 \\
Education & & & \\
$\quad$ Elementary school & 3.56 & 3.40 & 1.93 \\
$\quad$ Middle school & 4.33 & 1.75 & $5.16 \dagger$ \\
$\quad$ Above high school & 1.96 & 2.12 & \\
\hline
\end{tabular}

* Data from the 1998 National Health and Nutrition Survey ${ }^{2}$.

† Above middle school.

Table 2, there is an important inverse relationship between obesity and income and education in South Korea ${ }^{2}$.

The prevalence of obesity is not only much lower than in Western countries, but also lower than that of other Asian countries ${ }^{1}$. However, overweight seems to be one of the major public health issues in South Korea.

\section{Changes in disease patterns}

A longer life expectancy has resulted in the growth of the elderly population. The percentage of the elderly population, over the age of 65 years, was $7.1 \%$ in Korea in 2000, and the National Statistical Office expects the percentage to increase to $14.3 \%$ of the total population in 2022 and over $20 \%$ in $2032^{4}$. The growth of the elderly population, along with changes in the environment including diet, has brought changes in disease patterns and influenced the causes of death.

National morbidity data first became available from the National Health and Nutrition Survey of 1998. Among the most frequent chronic conditions are arthritis, gastritis and hypertension. There is a high prevalence of hypertension, with a rate of $27.8 \%$ among those over the age of 30 years (25.2\% for women and $30.2 \%$ for men). This rate is comparable to that of Singapore ${ }^{7}$. Using a cut-off for fasting blood sugar level of $140 \mathrm{mg} \mathrm{dl}^{-1}$ to define diabetes, $13 \%$ of men over 50 years of age and $10 \%$ of women over the age of 60 suffer from diabetes. If a total cholesterol level over $240 \mathrm{mg} \mathrm{dl}^{-1}$ is defined as high risk, this applies to $10 \%$ of adults over 30 years of age. Among the same age group, $12.5 \%$ of men and $4.9 \%$ of women are hypertriglyceridaemic (over $\left.210 \mathrm{mg} \mathrm{dl}^{-1}\right)^{2}$.

In Korea, a transition in the cause of death from communicable to non-communicable diseases is estimated to have occurred around 1970, compared with 1940 for the United States and 1950 for Japan ${ }^{8}$. The rate of mortality (per 100000 of the population) from infectious and parasitic diseases in 1985 was 23.2 (Fig. 2); in 1999, this decreased to 11.7. On the other hand, the rate from malignant neoplasm grew steadily from 88.3 in 1985 to 116.6 in 1999. An examination of deaths caused by sitespecific cancers shows that cancers of the lung, colon and breast are rapidly increasing as causes of death. The rate of mortality from diseases of the circulatory system dropped from 180.4 in 1985 to 122.0 in 1999 , but is still the primary cause of death ${ }^{4}$. Cerebrovascular disease accounts for a large portion of deaths caused by diseases of the circulatory system. The percentage of deaths caused by hypertensive diseases is decreasing, whereas death by ischaemic heart disease is rising ${ }^{4}$.

\section{Discussion}

The economic changes in South Korea and the concomitant changes in diet between World War II and the new millennium have been very large. After hosting the Olympics in 1988, South Korea was immersed in waves of internationalisation, leading to a steady increase in chronic diseases caused by industrialisation, pollution, prolongation of average life expectancy and changes in lifestyle, including eating habits. Entering the 1990s, many Korean scholars warned of the dangers of increased obesity and chronic diseases caused by a shift from the traditional diet to a Westernised diet of greater animal foods and fat intake, and insisted that a national nutrition policy was badly needed ${ }^{9-13}$.

The most noticeable feature of the South Korean nutrition transition is that the dietary shift has not been linked with a level of fat intake commensurate with its income level ${ }^{1}$. The relationship between gross national product (GNP) per capita and dietary fat intake was studied for 121 countries $^{14}$. From these studies, we expected that the proportion of energy from fat in South Korea in 1996 would be $35.5 \%$. The actual percentage of energy from fat was 16.7 percentage points less than this expected level ${ }^{1}$. The main focus of the discussion is how this uniqueness could have been retained in South Korea through nutrition policies and programmes, even with rapid economic growth, since an increase in energy from fat seems to be a result of a Westernisation of diets in many other countries.

\section{Why is dietary fat intake so low and vegetable intake so bigh in South Korea?}

The traditional Korean diet is a low-fat and high-vegetable $\operatorname{diet}^{12,13,15-17}$. Therefore, nutrition specialists are initiating numerous efforts to advertise and teach the public that the traditional diet is a healthy diet and that Western eating habits can be dangerous. They are also working on revival of the traditional diet using an approach that is acceptable to contemporary Koreans ${ }^{16,17}$.

Reasons for the low fat intake in South Korea suggested earlier by Kim et al. are the still relatively high level of 


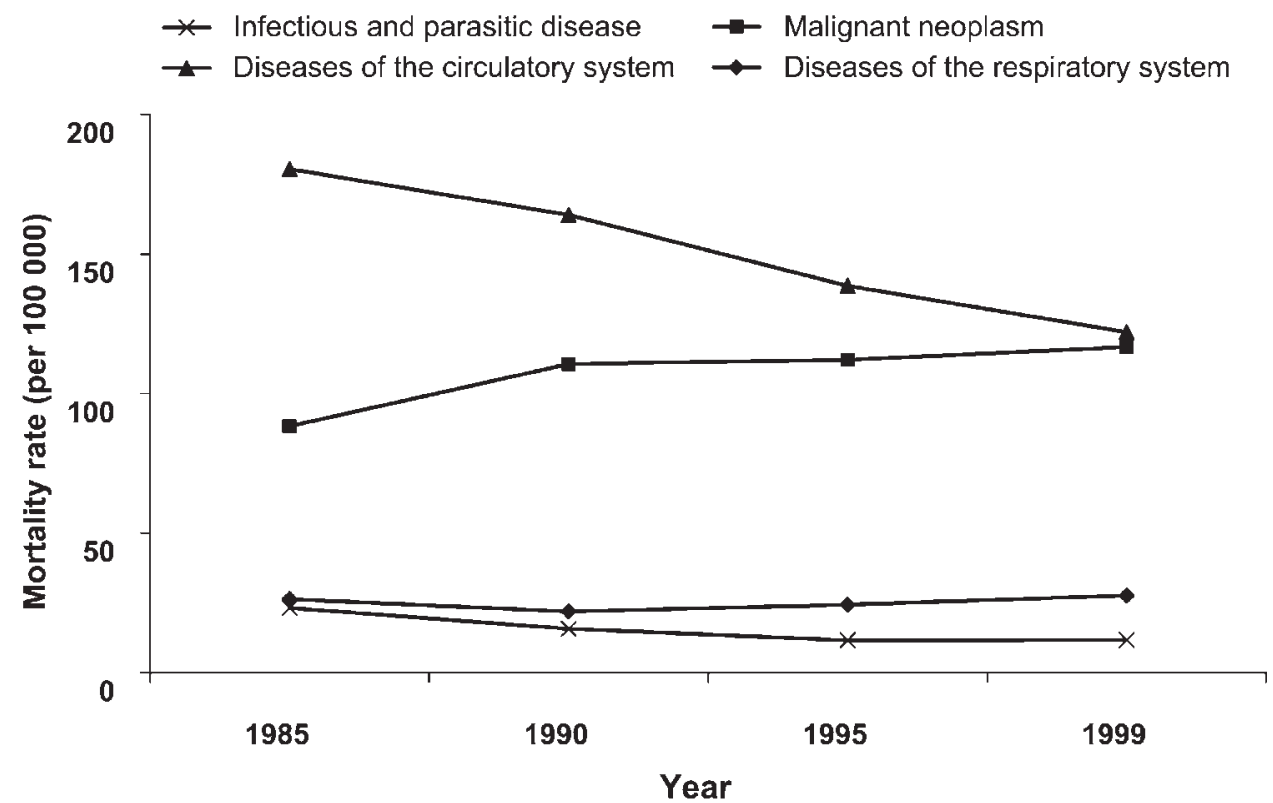

Fig. 2 Trends in the rates of mortality in South Korea ${ }^{4}$

carbohydrate intake, the style of cooking and, most importantly, the various movements to retain the traditional diet ${ }^{1}$. Contrasting to the low level of fat intake, vegetable consumption in South Korea is among the highest in Asia $^{5}$.

Commonly used vegetables in Korea are very diverse: wild edible greens, such as Chinese bellflower and bracken; field-grown greens, such as shepherd's purse and wild garlic; and cultivated vegetables, such as eggplant, squash and cucumber. It is reported that over 300 types of vegetable are eaten in rural areas. Vegetable foods are deeply related to the traditional Korean dietary culture. Vegetables are used in so many dishes - such as rice steamed with vegetables, porridge, rice cakes, soup, Kimchi, casseroles, steamed vegetable dishes, Na-mul (cooked vegetables), Sang-chae (raw vegetables), Ssam (vegetable wrap), fried vegetables, hard boiled food, and so on - that it is essentially true that there is no Korean dish that does not have some vegetables in it. This shows the important role vegetables play in the Korean diet. Because Koreans have enjoyed vegetables for such a long time, processing of vegetables has been advanced. The most typical processed vegetable food in South Korea is Kimchi, the main subsidiary food of the Korean diet. There are over 140 kinds of Kimchi and it can also be used as an ingredient for various dishes, such as Kimchi stew and Kimchi pancakes ${ }^{15-17}$. It has been observed that the consumption of Kimchi has been decreasing among the younger generation recently, but Kimchi still remains the most consumed food after rice, the staple food, and accounts for approximately $40 \%$ of the total vegetable intake $^{2}$. Also, even though meat consumption is increasing, the consumption of meat cooked in the Korean style is still higher than that of meat cooked in a Western style. The results of the 1993 National Nutrition Survey showed that the mainly consumed types of meat are found in soups, Bul-go-gi (grilled marinated beef) and grilled pork belly ${ }^{18}$. It is important to note that these meat dishes are usually consumed with vegetables, typically wrapped in various types of leaves (Ssam). Therefore, the increase in meat intake does not necessarily mean an increase in Westernstyle meat dishes or a decrease in vegetable intake.

The main reason why the fat intake does not skyrocket and the vegetable consumption level remains higher than that of other countries is because the vegetable-centred traditional dietary culture is still dominant in South Korea. Korean society has undergone vast changes in the consumption of clothing, food and housing due to industrialisation. However, its dietary habits have remained relatively intact despite the influx of changes, due to the conservative attitude of Koreans towards food. There has also been a recent boom in the demand for traditional restaurants in South Korea. Restaurants called 'Country dining table' that serve mostly vegetable side dishes, 'Raw vegetable house' where people use assorted vegetables to wrap their own food, and 'Native local food restaurants' and 'Buddhist temple restaurants' where meats are excluded, are gaining popularity, even among modernised people. The trend of pursuing healthy foods had already begun in the mid-1980s when chronic illnesses became a major health concern in South Korea. This trend reflects efforts to solve health problems by improving the diet.

Nutrition policies and programmes related to the retention of the traditional diet in South Korea

Concern on the part of the government, scholars and citizens about health and efforts to protect rural and local 
economies also played a large role in conservation of the traditional Korean diet. After local autonomy was in effect in South Korea, self-governing bodies publicised and conserved native local foods and developed dishes using local agricultural products to trigger local economic growth and protect local farming. Local governments held public events and promoted public relations to do this, promoting the vegetable-centred traditional diet in the process.

Publicity and education through diverse channels were important factors in helping people become aware that the traditional Korean diet, which is high in vegetables and low in fat, is a healthy diet. The role of the government in training housewives and other national initiatives from the government were discussed elsewhere ${ }^{1}$. By analysing articles related to eating habits and food consumption patterns in Korean newspapers after the 1960s, we observed that articles on diet increased in the 1980s, showing a growing interest in nutrition. In the 1960s and 1970s, most articles were related to food sanitation and policies, whereas after the 1980s, articles on nutrition, health and disease increased greatly, especially in the 1990s; this reflects an increased interest in health even in a public with improved economic status ${ }^{19}$. The Korean Dietetic Association (KDA) is one of the private organisations that have been actively involved in various nutrition intervention activities in South Korea. It was established in 1969 and has 85000 dietitian members. The main health promotion services of the KDA are aiding nutrition services at community health centres, providing nutrition education for national health, holding seminars and obesity camps, supplying nutrition information for citizens through their Internet homepage, monitoring food and nutrition information in the mass media, and holding national nutrition campaigns. They also produce and distribute various educational materials on nutrition. As part of an effort of public education in nutrition, the KDA held 'The first exhibition of nutritionally balanced diet for health' in 1982 and have conducted nutritional education for industrial workers since 1986. During the 1990s they undertook various activities to promote a more healthful traditional diet, such as running a campaign for the conservation of traditional Korean food culture in 1991, supporting the nutrition services of test community health centres nation-wide and monitoring food/nutrition information in the mass media since 1994. They have also provided information on traditional diets, helping to spread the knowledge that traditional food is healthy food. During the campaign about obesity, they publicised the fact that the traditional menu was advantageous in fighting obesity because the traditional Korean diet was a low-fat and high-vegetable diet. They also offered a traditional menu using native crops in order to revive traditional dietary culture during a nutrition campaign. Not only did they offer a variety of traditional menus to elementary schools, they also sent written messages about preserving the traditional dietary culture to students' homes and held lectures for parents ${ }^{20,21}$.

In summary, a range of government, nutrition specialists and some private organisation efforts have worked to retain healthful dimensions of the traditional diet in South Korea. The continued low level of total fat in the overall diet and the high intake of fruit and vegetables (a large proportion of which comes from Kimchi) bode well for South Korea. Clearly, these dietary patterns are related to the low obesity levels. South Korea has a remarkably low level of adult obesity; however, overweight is a major public health issue. Many studies have shown that overweight and obesity usually accompany higher prevalences of hypertension, diabetes and other noncommunicable chronic diseases (NCDs). People with a BMI between 25 and $30 \mathrm{~kg} \mathrm{~m}^{-2}$ in South Korea often suffer from obesity-related diseases. Taking these facts into consideration, keeping a lower BMI and seeking the suitable cut-off point for overweight and obesity in South Korea might be very important. Further understanding of the approaches used in South Korea to retain this diet, activity and body composition pattern will be important for South Korea, as well as other countries now undergoing this rapid nutrition transition.

\section{References}

1 Kim SW, Moon SJ, Popkin BM. The nutrition transition in South Korea. Am. J. Clin. Nutr. 2000; 71: 44-53.

2 South Korean Ministry of Health and Welfare. Reports on 1969-95 National Nutrition Survey; Report on 1998 National Health and Nutrition Survey. Seoul, South Korea: Ministry of Health and Welfare, 1969-99 [in Korean].

3 International Obesity Task Force. Obesity: Preventing and Managing the Global Epidemic. Report of a WHO consultation on obesity. Geneva: World Health Organization (WHO), 1998.

4 South Korean National Statistical Office. Annual Report on Cause of Death Statistics. Seoul, South Korea: National Statistical Office, 1999 [in Korean].

5 Kim SW, Popkin BM. Reply to salt consumption during the nutrition transition in South Korea [letters to the editor]. Am. J. Clin. Nutr. 2000; 72: 199-201.

6 Park MA, Lee HS, Kye SH, Moon HK. Study for major nutrients sources of foods by Korean Nutrition Survey energy, protein, fat, carbohydrate and crude fiber. Korean J. Nutr. 1997; 30: 84-90 [in Korean].

7 Singapore Ministry of Health. National Health Survey 1998. Singapore: Epidemiology \& Disease Control Department, Ministry of Health, 1998.

8 Drewnowski A, Popkin BM. The nutrition transition: new trends in the global diet. Nutr. Rev. 1997; 55: 31-43.

9 Moon SJ. Nutrition problems of Koreans. Korean J. Nutr. 1996; 29: 371-80 [in Korean].

10 Lee HK. Nutritional problems of Koreans: pattern of disease incidence and nutrition in Korea. Korean J. Nutr. 1996; 29: $381-3$ [in Korean].

11 Lee IH. Changes in dietary patterns and health status in Korea. Korean J. Dietary Culture 1993; 8: 359-72 [in Korean].

12 Lee KY, ed. The Evaluation of the Korean Diet: Focusing on the 20th Century. Seoul, South Korea: Shin-Kwang Publishing Co., 1998 [in Korean]. 
13 Paik HY, Moon HK, Chil YS, Ahn YO, Lee HK, Lee SW. Dietary Life and Disease of Koreans. Seoul, South Korea: Seoul National University Press, 1997 [in Korean].

14 Guo X, Mroz TA, Popkin BM, Zhai F. Structural changes in the impact of income on food consumption in China, 198993. Econ. Dev. Cult. Change 2000; 48: 737-60.

$15 \mathrm{Kim}$ SH, Oh SY. Cultural and nutritional aspects of traditional Korean diet. World Rev. Nutr. Diet. 1996; 79: 109-32.

16 Chang YJ. A strategic approach to commercialize traditional Korean foods. Food Industry Nutr. 1999; 4: 35-9 [in Korean].

17 Kim SH, Chang MJ, Cho MS, Chung HK, Oh SY, Chang YA. The Cultural Understanding of Dietary Life. Seoul, South Korea: Shin-Kwang Publishing Co., 1999 [in Korean].
18 Kye SH, Lee HS, Park MA, Moon HK. The study on frequently consumed food items from 1993 Korean National Nutrition Survey (II) - amounts and frequency of dishes intakes. Korean J. Dietary Culture 1996; 11: 581-92 [in Korean].

19 Kim EK, Park TS, Park YS, Jang MR, Lee KW. The content analysis of food and nutrition articles in the Korean newspapers - from January 1960 to June 1996. Korean J. Dietary Culture 1996; 11: 527-38 [in Korean].

20 The Ministry of Health and Welfare [Online]. Available at http://www.mohw.go.kr. Accessed May 2001.

21 The Korean Dietetic Association (KDA) [Online]. Available at http://www.dietitian.or.kr. Accessed May 2001. 